\title{
Assessment of Dislocation Density by Various Techniques in Cold Rolled 1050 Aluminum Alloy
}

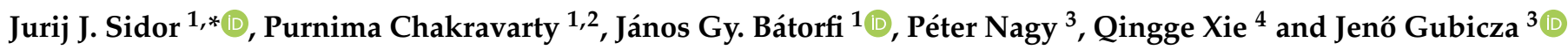 \\ 1 Savaria Institute of Technology, Faculty of Informatics, Eötvös Loránd University (ELTE), Károlyi Gáspár tér 4, \\ 9700 Szombathely, Hungary; pc@inf.elte.hu (P.C.); bj@inf.elte.hu (J.G.B.) \\ 2 Doctoral School of Physics, Faculty of Natural Sciences, Eötvös Loránd University (ELTE), P.O. Box 32, \\ 1518 Budapest, Hungary \\ 3 Department of Materials Physics, Faculty of Natural Sciences, Eötvös Loránd University (ELTE), P.O. Box 32, \\ 1518 Budapest, Hungary; nagyp@student.elte.hu (P.N.); gubicza@metal.elte.hu (J.G.) \\ 4 Collaborative Innovation Center of Steel Technology, University of Science and Technology Beijing, \\ Beijing 100083, China; xqg1084@126.com \\ * Correspondence: js@inf.elte.hu
}

Citation: Sidor, J.J.; Chakravarty, P.; Bátorfi, J.G.; Nagy, P.; Xie, Q.;

Gubicza, J. Assessment of Dislocation Density by Various Techniques in Cold Rolled 1050 Aluminum Alloy. Metals 2021, 11, 1571. https:// doi.org/10.3390/met11101571

Academic Editor: Alberto Moreira Jorge Junior

Received: 24 August 2021

Accepted: 27 September 2021

Published: 30 September 2021

Publisher's Note: MDPI stays neutral with regard to jurisdictional claims in published maps and institutional affiliations.

Copyright: (c) 2021 by the authors. Licensee MDPI, Basel, Switzerland. This article is an open access article distributed under the terms and conditions of the Creative Commons Attribution (CC BY) license (https:// creativecommons.org/licenses/by/ $4.0 /)$.

\begin{abstract}
This study examines the evolution of dislocation density in cold rolled 1050 Al alloy. Various techniques such as numerical approaches, indentation techniques, X-ray diffraction line profile analysis, and electron backscattering diffraction were employed for the characterization of the deformed state. These methods allowed us to determine the nature of the evolution of the dislocation substructure during cold rolling. The investigated material was subjected to thickness reductions varying from $5 \%$ to $47 \%$, which resulted in a continuous increase in hardness while the estimated dislocation density showed a tendency towards a less intense increase after a $\sim 30 \%$ straining level. The numerical approaches employed, such as the Kubin-Estrin and a modified version of this model, are capable of ensuring a reasonable estimation of dislocation density at low and moderate deformation levels $(\sim 5-30 \%)$, while the discrepancy between the measured and simulated data is negligible when the material has been exposed to more severe rolling reductions.
\end{abstract}

Keywords: dislocation density; Al alloys; X-ray line profile analysis; microhardness

\section{Introduction}

The extraordinary material properties of aluminum alloys, such as their strength to weight ratio, ductile nature, cryo-tolerance, corrosion resistance, and high energy absorption capacity, make them outstanding candidates in terms of their applicability in a wide range of products. In numerous cases, $\mathrm{Al}$ alloys are used in the form of flat products, the production of which involves a sequence of heat treatment and deformation processes or a combination of both. Throughout the thermomechanical processing of rolled sheets, the polycrystalline aggregate experiences morphological changes on the microscopic level, whereas the specific crystal lattice planes slip along glide planes on the nano-scale [1-4]. For FCC materials with a high stacking fault energy (SFE), this mechanism of deformation induces the generation of one-dimensional lattice defects [4], known as dislocations, which tend to block the slippage of each other, and thus resist deformation [2,3]. In both hot and cold rolling, the formation of dislocations takes place, while the annihilation of dislocations occurs during the process of annealing [4]. In cold rolling, deformation tends to break and offset the lattice planes, which increases the number of dislocations [1]. On the macroscopic level, the accumulation of dislocations enhances the hardness in the deformed metal, whereas during annealing the dislocation structure plays an important role from the point of view of morphological changes such as grain structure and crystallographic texture $[4,5]$. Given this, the assessment of substructure evolution in metallic systems is of crucial importance. 
Both direct and indirect techniques are employed for the estimation of dislocation density. Typically, the number of one-dimensional defects is determined using transmission electron microscopy (TEM), which provides a direct image of the substructure that has evolved. As an alternative to TEM, the quantity of dislocations can also be evaluated indirectly by X-ray diffraction line profile analysis (XLPA) [6-8], indentation techniques [9] or electron backscattering diffraction (EBSD) [10]. In XLPA, the data are obtained in the form of diffraction lines and the parameters of the defect structure are extracted by analyzing the width and shape of the diffraction peak profiles [6-8]. The advantages of XLPA over TEM are related to the field of view (bulk in XLPA and very local in TEM) and the possibility of choosing any arbitrary area. The X-ray line profiles are usually broadened due to the finite crystallite size and the lattice defects, such as dislocations. The whole line profile can be obtained as the convolution of the peak components related to the crystallite size and dislocations. Thus, the Fourier transform of the line profile is the product of the Fourier transforms of the size and dislocation peak profile components, denoted by $\mathrm{A}^{\mathrm{S}}$ and $\mathrm{A}^{\mathrm{D}}$, respectively. This product can be expressed as [11-13]:

$$
A(L)=A^{s} A^{D}=A^{s} \exp \left[\frac{1}{2}\left(-\pi b^{2}\right) \rho L^{2}\left(g^{2} C\right) f(\eta)\right]
$$

where $g$ defines the magnitude of the diffraction vector, $b$ is the Burgers vector of a dislocation, $\rho$ is the average dislocation density, $C$ is the average dislocation contrast factor, $\eta=$ $\mathrm{L} / \operatorname{Re}(\operatorname{Re}$ is the effective outer cut-off radius of dislocations), $\mathrm{L}$ is the Fourier length [11-13]. $\mathrm{A}^{\mathrm{S}}$ is a function of $\mathrm{L}$ as well as the median and the variance of the crystallite size distribution function. The $f(\eta)$ function in Equation (1) is known as Wilkens' strain function and is valid for dislocations in the entire L range $(0 \leq \mathrm{L} \leq \infty)$ [14]. A reliable evaluation of the X-ray diffraction patterns based on Equation (1) is the so-called convolutional multiple whole profile (CMWP) fitting method. In this procedure, the diffraction pattern is fitted by the sum of a background spline and the calculated intensity profiles for all available peaks where each peak profile is obtained as the convolution of the size and dislocation peak profiles, i.e., practically as the inverse Fourier transform of Equation (1) [14]. In addition, an experimentally determined instrumental profile is also convoluted to each calculated peak used for fitting. In the CMWP analysis, the calculated pattern depends on two parameters of the assumed log-normal crystallite size distribution (the median and the log-normal variance) and three parameters of the dislocation structure for face-centered cubic crystals $(\rho$, Re and a parameter characterizing the contrast factor $C)$. The pattern fitting results in these parameters of the microstructure.

Indentation techniques are a useful tool for the study of dislocations due to their ability to assess the evolution of crystal imperfections over a larger area $\left(\sim \mathrm{mm}^{2}\right.$ or even larger) [9,15-18]. By approximating the flow stress $\sigma_{y}$ from the Vickers hardness $H_{v}\left(\sigma_{y}\right.$ $\approx H_{v} / 3.06$ [19]), the stored energy $\left(E_{D}\right)$, caused by the presence of dislocations, can be estimated from the measured $H_{v}$ values via the following relationship $[9,15]$ :

$$
E_{D}=\frac{H_{v}^{2}}{G[3.06 M \alpha]^{2}}
$$

where $M$ stands for the Taylor factor, $\alpha$ is the geometric constant $(\sim 0.5)$ and $G$ is the shear modulus.

The stored energy and the dislocation density $\rho$ are related to each other as [4]:

$$
E_{D}=\alpha \rho G b^{2}
$$

By combining Equations (2) and (3), one can estimate the dislocation density by using Equation (4), although it should be mentioned that this is an approximative method.

$$
\rho=\frac{1}{\alpha^{3}}\left(\frac{H_{v}}{3.06 M G b}\right)^{2}
$$


The total dislocation density evolved in deformed materials consists of two parts: (i) geometrically necessary dislocations (GNDs), and (ii) statistically stored dislocations (SSDs). The GNDs appear due to the geometrical constraints encountered by the system, while the SSDs evolve from random trapping processes during plastic deformation [3]. The GNDs can be calculated from the EBSD data by the determination of the lattice curvature induced Nye's tensor component fields using orientation maps or by computing Kernel average misorientation [10,20-23]. Examination of GNDs via orientation imaging microscopy reveals the heterogeneity of the dislocation density throughout the volume of the material [20-23].

There have been many attempts to estimate the evolution of dislocations by employing analytical or empirical approaches. The original Williamson-Smallman method [24,25] was used for the assessment of $\rho$ during severe plastic deformation and strains of moderate value [26-28]. This approach suggests that the dislocation density is a function of crystallite size $D$ and microstrain $\varepsilon_{m}$ :

$$
\rho=\frac{3 \sqrt{2} \varepsilon_{m}}{D b}
$$

It should be noted that the Williamson-Smallman method cannot be used for the precise determination of the dislocation density since Equation (5) is only valid for a special arrangement of dislocations. In addition, the microstrain caused by dislocations is not a single value, but rather, depends on the indices of reflections due to the strain anisotropy of dislocations [8], which limits the reliable application of Equation (5). Furthermore, the correlation between the macroscopically imposed strain and the microstrain is not straightforward and this also complicates the applicability of the above-mentioned expression. As an alternative to the Williamson-Smallman method, the analytical approach developed by Csanádi et al. enables the computation of dislocation density as a function of macroscopically applied strain $\varepsilon[29,30]$ :

$$
\rho(\varepsilon)=\frac{2 C_{1}}{C_{4}}-\left(\frac{2 C_{1}}{C_{4}}-\rho_{0}\right)\left(1+\frac{C_{4} \varepsilon}{2}\right) \exp \left(-C_{4} \varepsilon\right)
$$

The model parameters $C_{1}$ and $C_{4}$ for aluminum are $2.33 \times 10^{14} \mathrm{~m}^{-2}$ and 1.15 , respectively [30].

The present work aims to reveal the evolution of dislocation density in cold rolled $1050 \mathrm{Al}$ alloy by using different methods in a comparative manner. Validation of different techniques can provide ideas for an efficient approach to the calculation of dislocation density in deformed materials under particular physical conditions.

\section{Materials and Methods}

The investigated $1050 \mathrm{Al}$ alloy (Al-0.3 wt\% Fe) was chosen because of its low solute content, and it was subjected to diverse cold rolling reductions. Prior to deformation, the initial material (sample A) was annealed at $550{ }^{\circ} \mathrm{C}$ to ensure a fully recrystallized state. Thickness reductions of 5.3\% (sample B), 15.8\% (sample C), $21.1 \%$ (sample D), $28.9 \%$ (sample E) and 46.8\% (sample F) were performed in one rolling pass at room temperature using laboratory rolling with a roll diameter of $150 \mathrm{~mm}$. Diverse straining levels guaranteed the evolution of various substructures in the rolled sheets B-F.

The development of microstructural features at different length scales was examined through X-ray diffraction, EBSD and indentation techniques. First, the investigated materials were exposed to X-ray diffraction line profile analysis, which allows the dislocation density to be calculated by analyzing the broadening of diffraction lines by the method described in great detail in [6,8]. Both annealed and deformed samples were studied on the plane perpendicular to the normal direction (ND plane). Mechanically grinded and polished samples were electropolished at a voltage of $20 \mathrm{~V}$ and current of $1 \mathrm{~A}$ for $8 \mathrm{~s}$ at room temperature using a solution of $70 \mathrm{vol}$ \% ethanol, 20 vol.\% perchloric acid and 10 vol.\% glycerin. The X-ray diffraction patterns were measured on the electropolished surfaces by a high-resolution rotating anode diffractometer (type: RA-MultiMax9, manufacturer: 
Rigaku, Tokyo, Japan) with CuK $\alpha 1$ radiation (wavelength, $\lambda=0.15406 \mathrm{~nm}$ ). The height and the width of the rectangular X-ray spot on the sample surface were 1.5 and $0.2 \mathrm{~mm}$, respectively. The peak profiles were evaluated by the CMWP fitting procedure [14]. The first nine reflections were used in the evaluation, which can be found at a diffraction angle

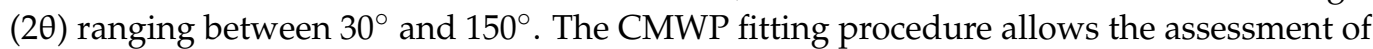
the dislocation density. More details about the XLPA evaluation can be found in [31].

The studied samples were also subjected to EBSD measurements across the thickness, which allows for fast data collection. In the case of the virgin sample (annealed sheet prior to rolling, sample A), the EBSD patterns were collected at a relatively high speed of $\sim 100$ frames per second (fpc), whereas the cold rolled sheets were measured at a data acquisition rate of $\sim 10 \mathrm{fpc}$. The EBSD was conducted on a plane perpendicular to the sample transverse direction (TD-plane). The orientation patterns were obtained by using the Hikari-type ${ }^{\circledR}$ detector (EDAX Inc., Mahwah, NJ, USA) attached to a high-resolution scanning electron microscope (FEG-SEM) FEI-TENEO ${ }^{\circledR}$ (Thermo Fisher Scientific, Brno, Czech Republic), whereas the post-processing was conducted with the commercial OIMTSL- $8{ }^{\circledR}$ software (EDAX Inc., Mahwah, NJ, USA). The annealed sample was subjected to orientation imaging microscopy (OIM) measurement at the acceleration voltage of $20 \mathrm{kV}$, while the pattern acquisition of deformed counterparts was performed at lower acceleration voltages $(17-19 \mathrm{kV})$ to avoid overlapping of the acquired patterns originating from the surface and deeper layers. In the SEM chamber, the examined samples were tilted $70^{\circ}$ with respect to the EBSD detector. The orientation distribution functions (ODFs) were displayed in the form of pole figures.

The sample preparation for OIM investigation was completed by the typical metallographic procedure developed for EBSD. This method includes mechanical grinding, polishing, and electrolytic polishing. The mechanical polishing was finished with two Struers ${ }^{\circledR}$-type DiaDuo suspensions (Struers, Ballerup, Denmark), which contain 3 and $1 \mu \mathrm{m}$ diamond particles, respectively. Afterward, the mirror quality surfaces were cleaned with liquid soap and rinsed with warm water and dried. The electrolytic polishing was conducted for 45-60 s at voltages ranging from 20 to $30 \mathrm{~V}$ with A2 Struers ${ }^{\circledR}$ electrolyte (Struers, Denmark). The A2 electrolyte was cooled to temperatures ranging between 0 and $5{ }^{\circ} \mathrm{C}$.

To investigate both the strain hardening phenomena and evolution of dislocation density, both annealed and deformed samples were subjected to indentation measurements, which were conducted by the Zwick/Roell ${ }^{\circledR}$ ZHV $\mu$-type Vickers microhardness tester (ZwickRoell, Brierley Hill, West Midlands, UK). The hardness measurements were carried out under loads ranging from $10 \mathrm{gf}$ to $2 \mathrm{kgf}$ by making square-shaped indents on the polished surfaces of the TD and ND planes.

The evolution of dislocation density was investigated on the ND-plane in the subsurface region inasmuch as the XLPA and indentation techniques require sample preparation, which involves the removal of $\sim 1 / 5$ th of the half-thickness from the surface layer.

\section{Results and Discussion}

\subsection{Evolution of Microstructure, Texture, and Hardness}

The evolution of microstructure during deformation was investigated by means of the orientation imaging contrast technique, which reveals both morphological changes in the grains involved in deformation and the development of orientation gradients in the crystals. Figure 1 indicates that the morphology of the deformed grains is correlated to the amount of deformation applied. After the $15.8 \%$ reduction, the majority of high angle grain boundaries are aligned along the rolling direction (RD). Since sample A was heat treated prior to cold rolling, the inverse pole figure (IPF) presented in Figure 1a shows either negligible orientation deviation within each grain or a few low angle grain boundaries in some instances. These microstructural features are characteristic of annealed materials. Figure $1 \mathrm{~b}-\mathrm{f}$ show the evolution of misoriented structures caused by cold rolling. It is obvious that increasing the degree of straining level induces grain fragmentation 
in the investigated materials (see Table 1). The development of orientation gradients in the grains of deformed materials B-F occurs simultaneously with the evolution of crystallographic texture (Figure 2) and generation of dislocations, that is, these processes are tightly connected to each other.

Table 1. Average $M$ values, grain sizes, and $e$ calculated investigated materials A-F.

\begin{tabular}{ccccc}
\hline Sample & Reduction $(\%)$ & Grain Size $(\boldsymbol{\mu m})$ & $\boldsymbol{M}$ & $\boldsymbol{e}$ \\
\hline A & 0 & 74.8 & 3.09 & 0 \\
B & 5.3 & 70.0 & 3.1 & 0.06 \\
C & 15.8 & 66.4 & 3.1 & 0.20 \\
D & 21.1 & 64.2 & 3.1 & 0.27 \\
E & 28.9 & 61.0 & 3.1 & 0.40 \\
F & 46.8 & 54.7 & 3.16 & 0.74 \\
\hline
\end{tabular}

Figure 2 shows the crystallographic aspects of the microstructural changes involved in deformation. Plane strain compression, as a major strain mode of rolling, causes reorientation of recrystallization (RX) texture components towards the deformation counterparts. In the present case, the cube-dominate texture of annealed sample A rotates to the socalled $\beta$-fiber (see Figures 2 and 3 for details). This complex-shaped fiber connects copper $\{112\}<111>$ and brass $\{011\}<112>$ via the S-component in Euler space, and the Miller indices of all orientations belonging to this fiber are described in detail by Sidor and Kestens [5].

Knowing the texture, one can compute the Taylor factor $M$ for each orientation and estimate the average value for the entire polycrystalline aggregate. Table 1 shows the calculated average Taylor factor for investigated materials A-F. This parameter is a measure of the crystallographically resolved hardness of a given orientation and it provides a guess of the hardening of textured metals. The average value of $M$ is necessary for the estimation of the dislocation density by employing Equation (4). It was already shown [32], that RX orientations have lower Taylor factors compared to deformation components, and therefore the tendency towards an increase in $M$ in rolled materials (see Table 1 ) can be explained by the enhancement in the fraction of rolling textures in the deformed materials B-F (see Figure 2).

The presence of dislocations in deformed polycrystalline aggregates accounts for strain hardening, since the flow stress as well as the hardness are proportional to the $\rho^{0.5}$ [4]. Figure 4 shows the strain hardening phenomenon caused by the thickness reduction in rolling. The equivalent strain $e$ (see Table 1 ) for the investigated subsurface region was calculated by means of the flow line model [33,34] in order to consider the amount of shear strain localized in this layer. It is obvious that a $5.3 \%$ thickness reduction leads to a considerable increase in hardness $\left(\mathrm{HV}_{0.5}\right)$, implying that the evolution of the substructure is intensive even at small straining levels. The hardening rate tends to decline after the $21.1 \%$ reduction and induces a slight increase in $\mathrm{HV}_{0.5}$. 
(a)

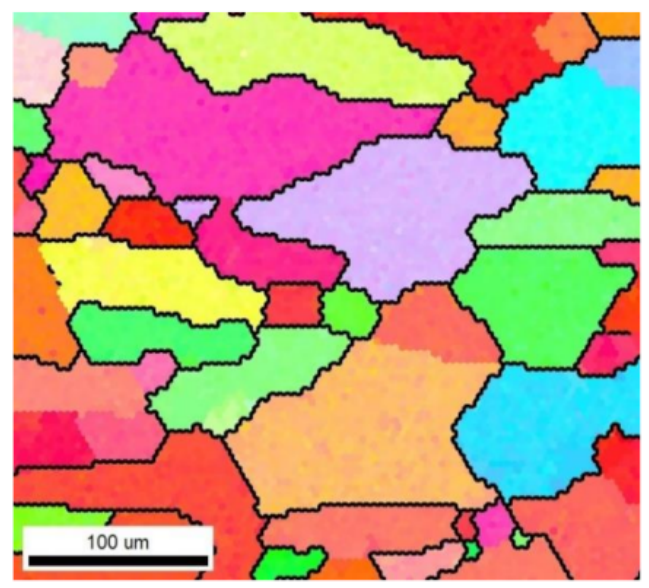

(c)

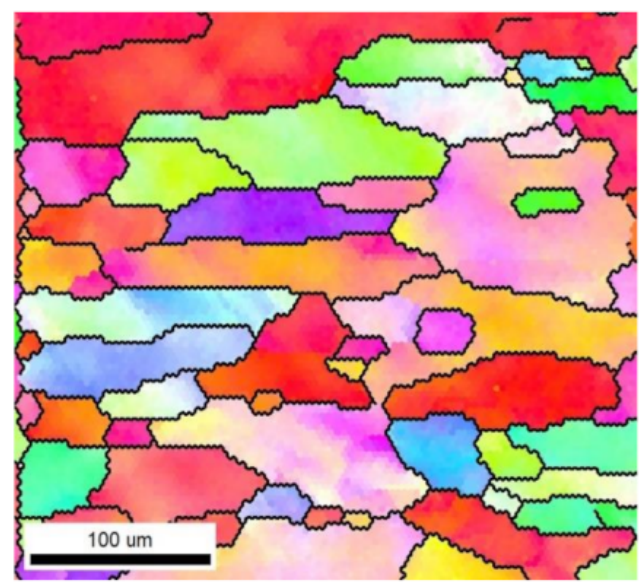

(e)

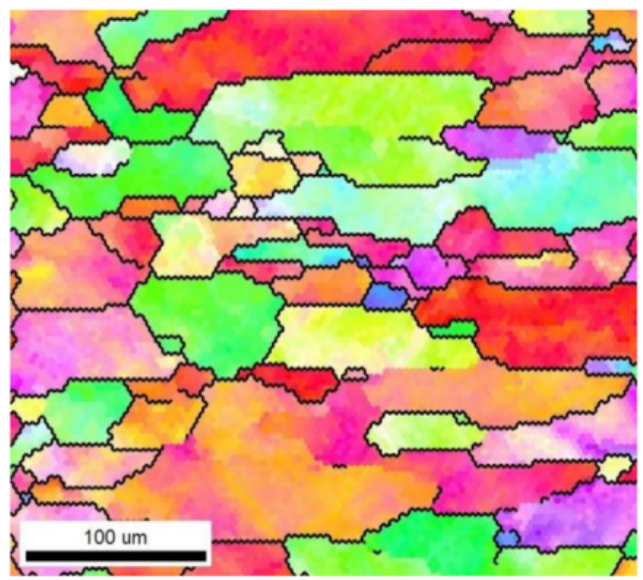

(b)

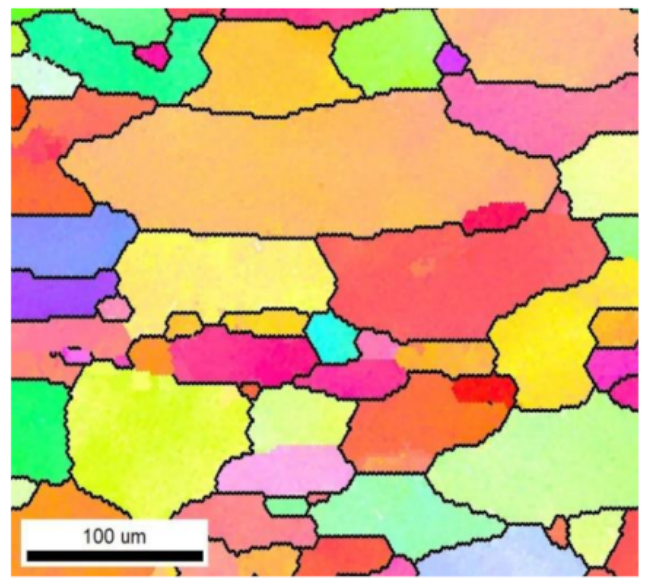

(d)
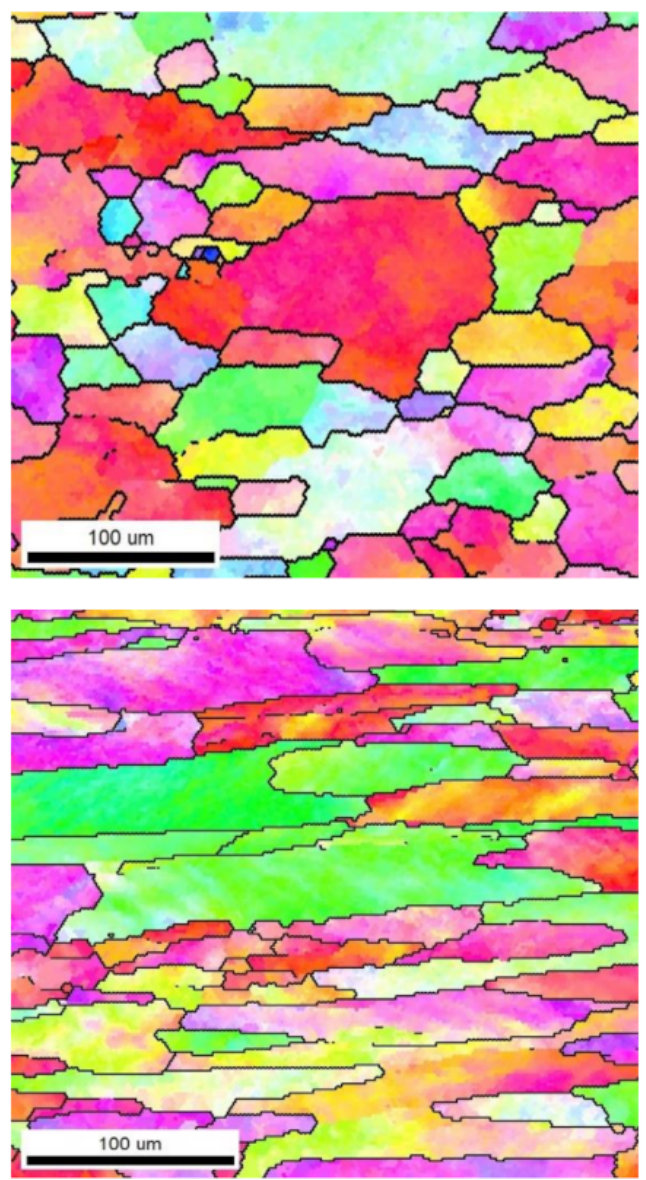

(f)

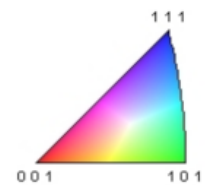

Figure 1. Inverse pole figure (IPF) maps measured on the transverse direction (TD) plane in the subsurface region after different rolling reductions by Electron Backscatter Diffraction (EBSD): (a) Sample A (0\% reduction); (b) Sample B (5.3\% reduction); (c) Sample C (15.8\% reduction); (d) Sample D (21.1\% reduction); (e) Sample E (28.9\% reduction); (f) Sample F (46.8\% reduction). 


\section{$\begin{array}{lllllll}1 & 1.2 & 1.6 & 2.02 & 2.5 & 3.2 & 4.1\end{array}$}

(a)

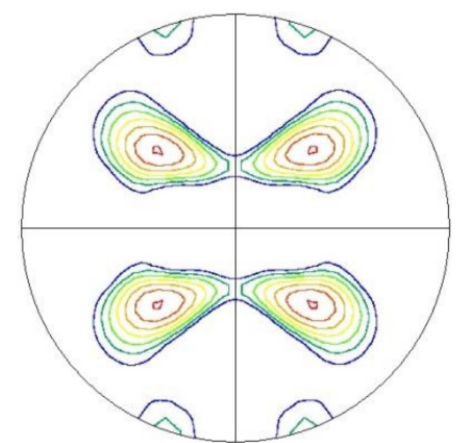

(c)

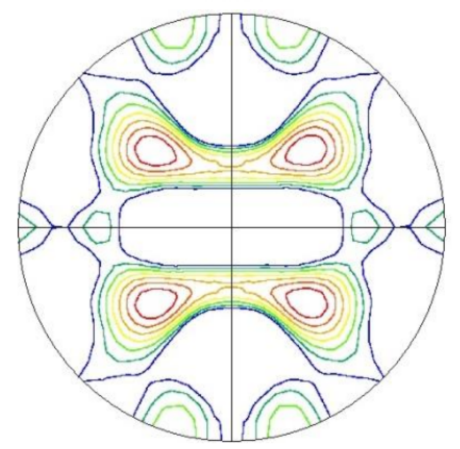

(e)

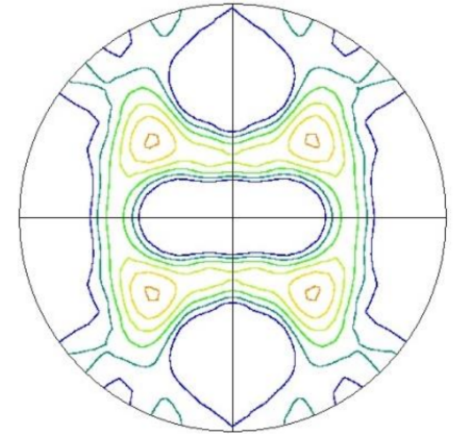

(b)

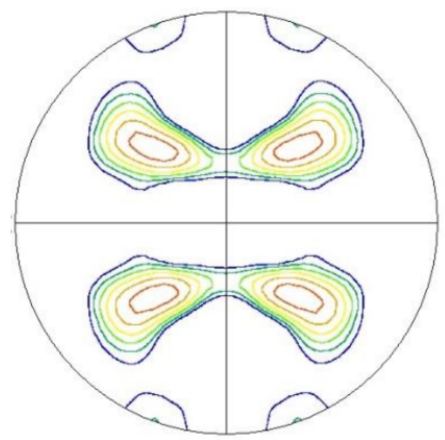

(d)

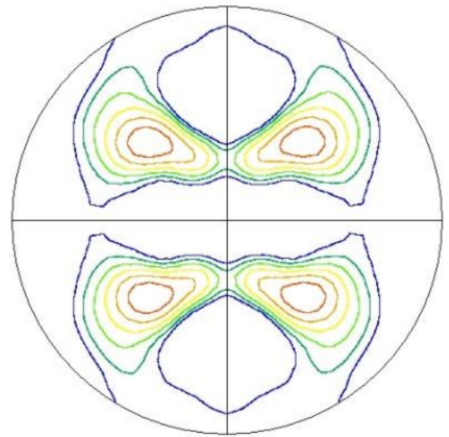

(f)

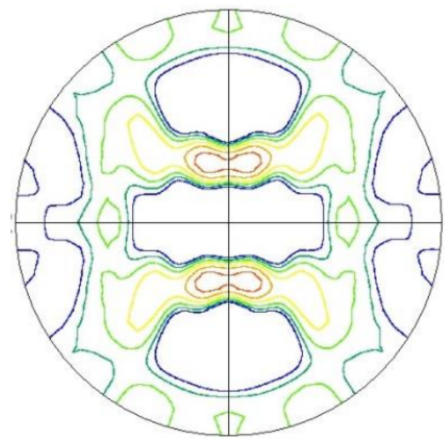

Figure 2. $\{111\}$ Pole figures measured by EBSD: (a) Sample A (0\% reduction); (b) Sample B (5.3\% reduction); (c) Sample C (15.8\% reduction); (d) Sample D (21.1\% reduction); (e) Sample E $(28.9 \%$ reduction); (f) Sample F ( $46.8 \%$ reduction).

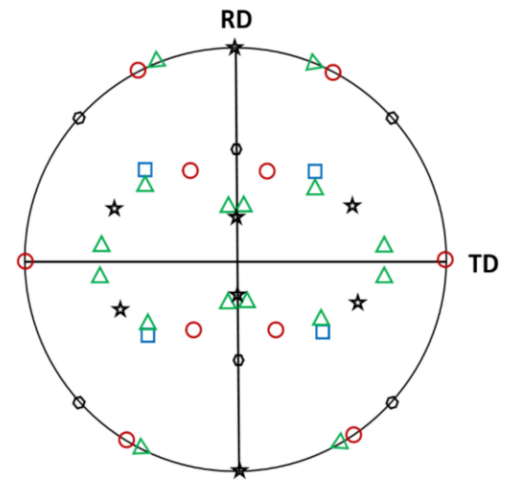

$\triangle S\{123\}<915$ 11>

O Brass $\{110\}<112>$

$\square \quad$ Cube $\{100\}<001>$

$\star$ Copper $\{112\}<111>$

D Goss $\{110\}<001>$

Figure 3. Key to $\{111\}$ Pole Figure, showing the major orientations that tended to evolve in the investigated samples. 


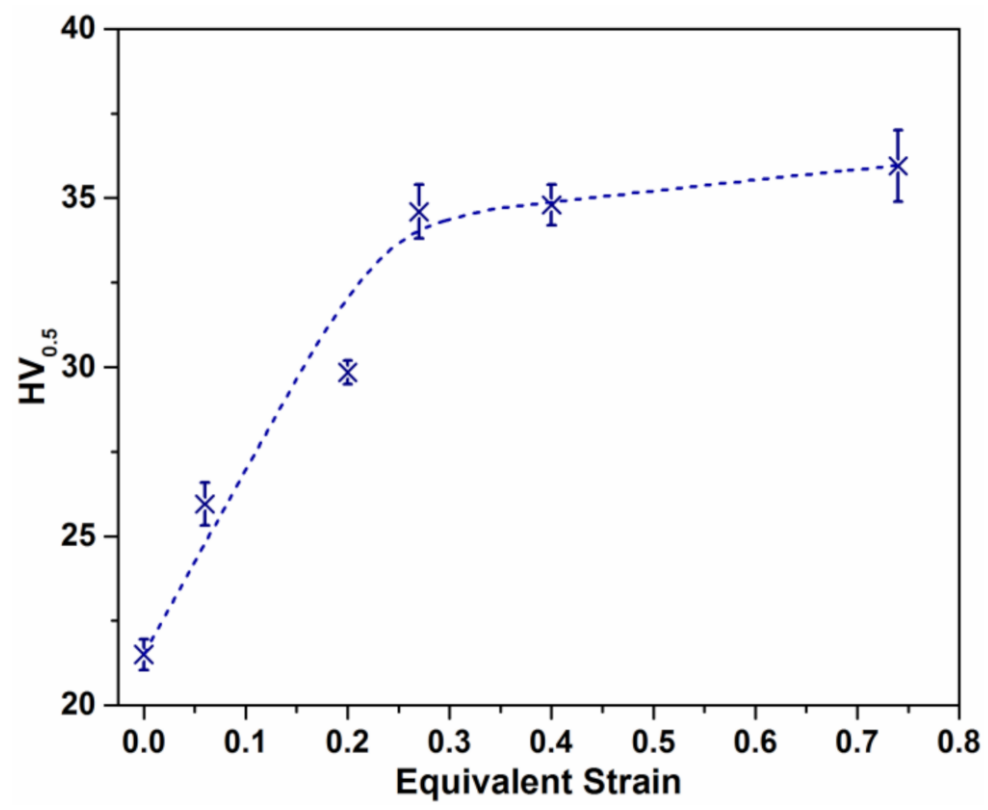

Figure 4. Change in hardness $\mathrm{HV}_{0.5}$ with strain, measured by microhardness tester with a load of $0.5 \mathrm{kgf}$.

\subsection{Estimation of Dislocation Density by XLPA}

A measure of the dislocation arrangements in polycrystalline aggregates can be performed by comparing the X-ray line profiles in deformation-free and strained materials. The presence of dislocations causes lattice distortions, and therefore the resulting X-ray diffraction lines tend to broaden (see Figures 5 and 6). By following the methods developed in $[6,8,14,35]$, it is possible to evaluate the measured diffraction profiles by the convolutional multiple whole profile fitting procedure so as to determine the dislocation density. As an example, Figure 5 shows the CMWP fitting for sample F rolled with a reduction of $46.8 \%$. Other examined samples revealed qualitatively identical diffraction patterns. It should be mentioned that for samples A (deformation free) and B (5.3\% reduction), the dislocation density was lower than the detection limit $\left(\sim 10^{13} \mathrm{~m}^{-2}\right)$ of the X-ray diffractometer employed. The dislocation density values computed by the XLPA are listed in Table 2. After the $15.8 \%$ reduction, the value of $\rho$ increased to $\sim 0.7 \times 10^{14} \mathrm{~m}^{-2}$ and was further enhanced by increasing the rolling reduction to $29 \%$. In this state, the $\rho$ was $\sim 1.2 \times 10^{14} \mathrm{~m}^{-2}$, whereas additional straining did not yield further increases in the dislocation density.

Table 2. Evolution of dislocation density as predicted by the X-ray Line Profile Analysis (XLPA) and average cell size approximated from the data of [36].

\begin{tabular}{ccc}
\hline Sample & $\boldsymbol{\rho} \times \mathbf{1 0}^{\mathbf{1 4}}\left(\mathbf{m}^{-\mathbf{2}}\right)$ & $\mathbf{d}(\boldsymbol{\mu m})$ \\
\hline A & $<0.1$ & - \\
B & $<0.1$ & 1.9 \\
C & $0.7 \pm 0.1$ & 1.1 \\
D & $0.9 \pm 0.1$ & 1 \\
E & $1.2 \pm 0.2$ & 0.9 \\
F & $1.2 \pm 0.2$ & 0.7 \\
\hline
\end{tabular}

Figure 6 reveals the effect of deformation on the broadening of $\{311\}$ diffraction peaks in the investigated materials. The degree of broadening increases with the amount of strain. The diversity between the undeformed sample A and one rolled with the 5.3\% (sample B) is negligible; thus, Figure 6 shows the reflection for the latter only. It is also obvious that the peak broadening for sample $\mathrm{F}$ ( $46.8 \%$ reduction) is more extensive as compared to sample E rolled with $28.9 \%$; however, the calculated dislocation densities in both materials 
seem to be identical within the experimental error. This effect can be explained by the dynamic equilibrium between the multiplication and annihilation of dislocations, resulting in a saturation of $\rho$. The difference in the breadth values of the $\{311\}$ diffraction peaks for samples $\mathrm{E}$ and $\mathrm{F}$ could be caused by the different arrangement of dislocations, since not only the density, but also the arrangement of dislocations influence the peak broadening. The crystallite size cannot be determined from the present XLPA measurements since the broadening of the peaks was suppressed by the instrumental effect. Thus, the average cell size d, was approximated from the dependence of d over the strain in $\mathrm{Al}$ alloys, as published in [36]. The cell size values are listed in Table 2. These values are higher than the detection limit of XLPA for the crystallite size in the present study (about $500 \mathrm{~nm}$ ), but it is apparent that the value of $\mathrm{d}$ follows a downwards trend with the increase in $e$. Although, the error of the cell size values was not determined, the difference between the cell size for samples $\mathrm{E}$ and $\mathrm{F}$ is clearly marginal, similar to the trend observed for the dislocation density.

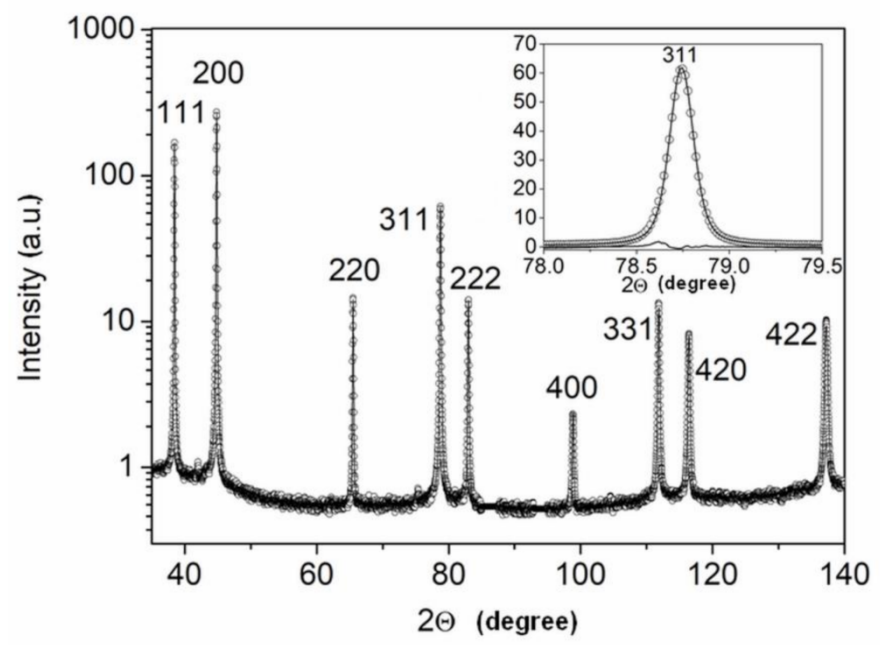

Figure 5. Measured X-Ray Diffraction (XRD) pattern (open circles) and corresponding diffractogram calculated by the convolutional multiple whole profile (CMWP) method (solid line) for the sample F rolled to $46.8 \%$ reduction. The magnified inset, corresponding to $\{311\}$ reflection, reveals the quality of the fitting. The difference between the measured and fitted data can be seen at the bottom of the inset.

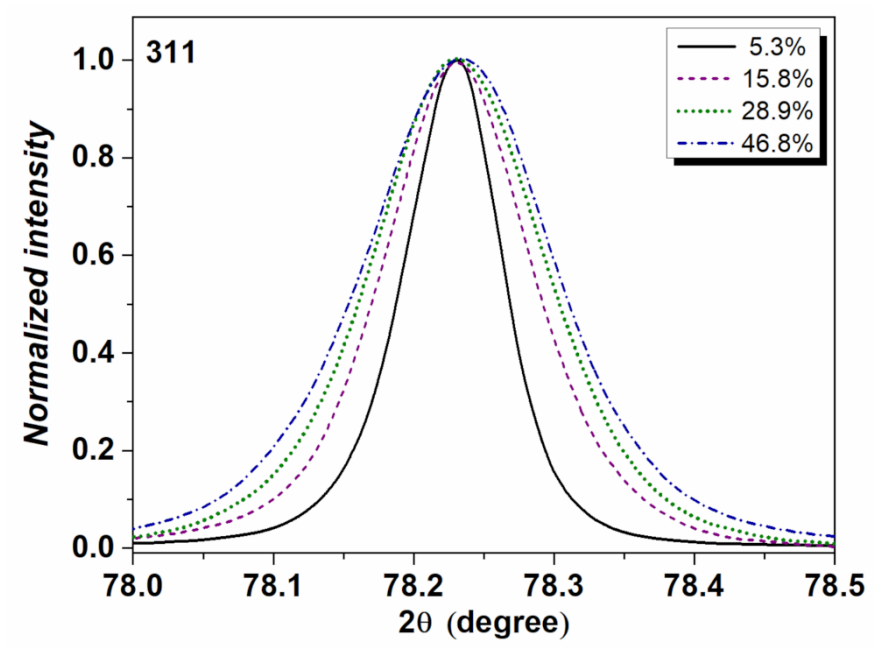

Figure 6. Broadening of $\{311\}$ reflection as a function of thickness reduction. 


\subsection{Indentation}

As shown in Figure 4, the hardening behavior of a material, which is related to the number of dislocations, can be indirectly quantified by indentation techniques. According to Equation (4), it is possible to follow the progress of the generation of dislocations by the measurements of the indentation hardness. In the current case, the Vickers indenter with square-based pyramidal geometry was forced onto the surfaces of the investigated samples with loads ranging from $10 \mathrm{gf}$ to $2 \mathrm{kgf}$. Knowing the geometry of the indenter, the diagonals, $d$ of the resulting square-shaped impression enable the calculation of the indentation depth, $h$. Figure 7 shows the dependence of the hardness (normalized by the saturation value $\mathrm{HV}_{\mathrm{s}}$ ) on indentation depth for a spectrum of loads used in the current study. In sample A (5.3\% thickness reduction), the hardness tends to decline in the range from $10 \mathrm{gf}$ to $100 \mathrm{gf}$ and becomes saturated $\left(\mathrm{HV}_{\mathrm{s}}\right)$ after applying a load of $200 \mathrm{gf}$. Other samples revealed similar evolutionary patterns of hardness variation over the indentation depths (see Figure 7); however, this effect becomes less pronounced with an increase in the deformation level and seems to vanish at $47.7 \%$ reduction. At smaller straining levels, we observe the so-called indentation size effect (ISE), when the hardness of a material is larger at lower scales compared to macroscopic one. Analysis of the ISE makes it possible to calculate the amount of GNDs and SSDs by using the Nix-Gao model [18], though this technique requires the application of loads lower than $10 \mathrm{gf}$ (generally nanoindentation is employed).

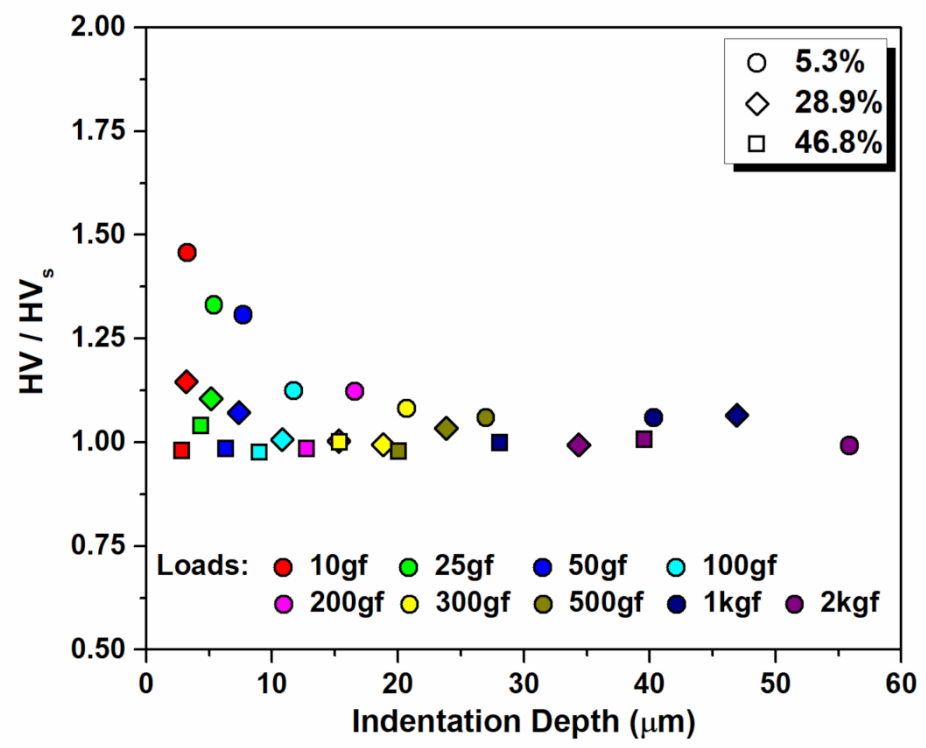

Figure 7. Variation in Vickers hardness with indentation depth after various straining levels. The $\mathrm{HV}_{\mathrm{S}}$ corresponds to the saturation level, i.e., when the hardness levels off.

In order to calculate the dislocation density, the geometric parameter $\alpha$ should be defined, which is generally considered to be independent of the degree of deformation; however, more accurate calculations show that this parameter may vary for the population of edge and screw dislocations for various materials subjected to a wide spectrum of straining, and can be estimated as [4]:

$$
\alpha \cong \frac{(1-0.5 v)}{4 \pi(1-v)} \ln \left(\frac{\rho^{-0.5}}{b}\right)
$$

where $v$ is a Poisson ratio (for $\mathrm{Al} v=0.35$ ).

Equation (7) suggests that the value of $\alpha$ depends on the dislocation arrangements and their quantity, since the mean free path of dislocations is inversely proportional to the $\rho^{0.5}$ [4]. As it also follows from Equation (7), the calculation of $\alpha$ requires defining the 
$\rho$, and because the latter is unknown, the assessment of dislocation density can be done by implementing numerical approximations, which are independent of $\alpha$. By employing Equation (6), one can approximate the value of $\rho(\varepsilon)$, and then compute the variation in $\alpha$ for a wide spectrum of reduction levels. Figure 8 shows the variation in $\alpha$ for different straining degrees, calculated by combining Equations (6) and (7). It is obvious that using commonly accepted $\alpha$ of $\approx 0.5$ is justified for high strains, whereas this parameter is higher for lower deformation levels. The decrease in $\alpha$ with the strain applied agrees with the increase in the average Taylor factor (Table 1), which is a dimensionless measure of the dissipated plastic power in a polycrystalline system. The Taylor factor can also be considered as the degree of the dislocation arrangement since it was shown that a system with a less clustered dislocation arrangement is characterized by the high $M$ value [37]. Such polycrystalline aggregates reveal a tendency to reduce dislocation hardening, which corresponds to a smaller value of $\alpha$.

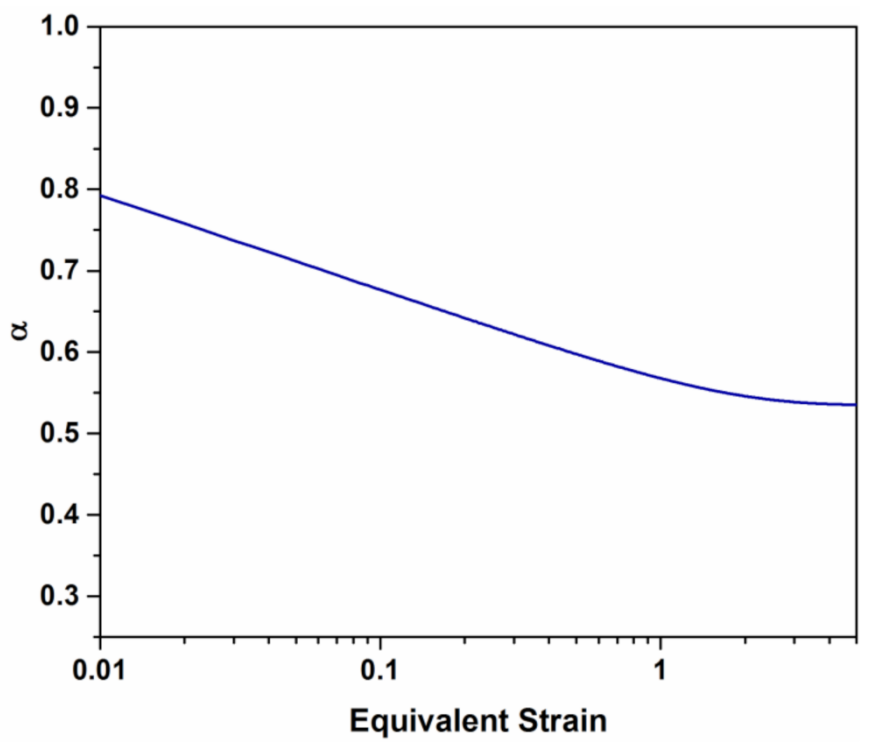

Figure 8. Dependence of $\alpha$ on the strain.

The calculation of $\rho$ with Equation (4) was performed by (i) using the Taylor factors listed in Table 1, (ii) considering HVs from the saturation zone (i.e., for loads above $100 \mathrm{gf}$ ), and (iii) computing $\alpha$ by Equation (7) (see Figure (8)). It should be noted that the value of $\mathrm{HV}$ in Equation (4) should be expressed in Pa. Table 3 reveals the estimated dislocation densities for the cold rolled materials B-F with the Burgers vector $b=0.286 \mathrm{~nm}$, and shear modulus $G=26 \mathrm{GPa}$. It is obvious that when the $\alpha=0.5$ was employed for all thickness reductions, the $\rho$ was significantly overestimated compared to the values obtained by the XLPA method (see Tables 2 and 3). In the case when the $\alpha$ was calculated by Equation (7), the dislocation densities estimated from the indentation experiments revealed good agreement with those measured by the $\mathrm{X}$-ray diffraction (Table 2), except of dislocation density, calculated for the 5.3\% $\left(\rho(\right.$ XLPA $)<0.1 \times 10^{14} \mathrm{~m}^{-2}$ and $\rho$ (Indentation $)=0.38 \times 10^{14}$ $\mathrm{m}^{-2}$ ). It seems that the assessment of $\rho$ by microhardness overestimates the dislocation density at lower deformation degrees. This effect may be caused by the development of additional dislocations during hardness testing. Indeed, deformation under the indenter can increase the dislocation density and this effect is more pronounced for lower strains when the material contains only a very low density of dislocations. Furthermore, estimation of the dislocation density in the unstrained sample A gives a value of $\sim 10^{13} \mathrm{~m}^{-2}$, which does not sound physically possible, and hence, the evaluation of $\rho$ by Equation (4) for very low strains (or unstrained materials) should be treated with particular care. 
Table 3. Evolution of dislocation density calculated by Equation (4) with various values of $\alpha$.

\begin{tabular}{ccc}
\hline Sample & $\boldsymbol{\rho} \times \mathbf{1 0}^{\mathbf{1 4}}\left(\mathbf{m}^{-\mathbf{2}}\right),(\boldsymbol{\alpha}=\mathbf{0 . 5})$ & $\boldsymbol{\rho} \times \mathbf{1 0}^{\mathbf{1 4}}\left(\mathbf{m}^{-\mathbf{2}}\right),(\boldsymbol{\alpha}$ by Equation $\mathbf{( 7 ) )}$ \\
\hline B & $1.02 \pm 0.13$ & $0.38 \pm 0.04$ \\
C & $1.47 \pm 0.07$ & $0.73 \pm 0.04$ \\
D & $1.76 \pm 0.11$ & $0.94 \pm 0.07$ \\
E & $1.99 \pm 0.07$ & $1.17 \pm 0.05$ \\
F & $2.25 \pm 0.51$ & $1.52 \pm 0.20$ \\
\hline
\end{tabular}

\subsection{Numerical Assesment of Dislocation Density}

When the polycrystalline system is subjected to plastic deformation, the formation of dislocations leads to strain hardening, as shown in Figure 4. Numerous studies [4,29-31,36,38] claim that the correlation between the flow stress $\sigma$ and dislocation density $\rho$ is described by the Taylor equation, where $\sigma$ is proportional to the $\sqrt{\rho}$. This relationship holds for both pure metals and alloys $[36,38]$. To address the problem of dislocation generation and stress saturation during plastic deformation, various models have been developed [29-31,36,38,39].

To assess the evolution of dislocations during deformation with high accuracy, it is necessary to consider the phenomena of dislocation formation and annihilation [36,3841]. Apart from this, the linear defects may interact with each other, leading to them being trapped. In view of this, researchers in this area have defined mobile $\rho_{\mathrm{m}}$ and forest $\rho_{\mathrm{f}}$ dislocations $[36,38,39]$, and the total dislocation density is composed of these two components. In our analysis, we employed the Kubin-Estrin (K-E) model [39], which requires a numerical solution for the following equations:

$$
\begin{gathered}
\frac{d \rho_{m}}{d \varepsilon}=C_{1}-C_{2} \rho_{m}-C_{3} \rho_{f}^{\frac{1}{2}} \\
\frac{d \rho_{f}}{d \varepsilon}=C_{2} \rho_{m}+C_{3} \rho_{f}^{\frac{1}{2}}-C_{4} \rho_{f}
\end{gathered}
$$

In the given approach, the model parameters $C_{i}$ are related to the competing mesoscopic processes, which tend to take place in the course of plastic deformation. The first two coefficients, $C_{1}$ and $C_{2}$ are responsible for the multiplication of mobile dislocations and their mutual trapping, whereas the immobilization via interaction with the forest dislocations is controlled by the $C_{3}$, while $C_{4}$ parameter governs the dynamic recovery. The following values of model parameters $C_{i}$ were defined for $\mathrm{Al}$ [30]: $C_{1}=2.33 \times 10^{14} \mathrm{~m}^{-2}$, $C_{2}=1.1, C_{3}=4 \times 10^{5} 1 / \mathrm{m}, C_{4}=1.2$. The K-E model requires the initial dislocation density, a characteristic of the unstrained material, which was approximated by the $\rho(\varepsilon=0)=10^{10} \mathrm{~m}^{-2}$, while the fraction of mobile and forest dislocations can be set to $\rho_{\mathrm{m}}=\rho_{\mathrm{f}}=\rho(\varepsilon=0) / 2[29,30]$. Figure 9 shows that the qualitative evolutionary pattern of dislocation density during rolling can be reasonably captured with the K-E model, while this approach also provides good agreement between the measured and simulated counterparts. For samples B-F, the predicted $\rho$ values are of the same order of magnitude compared to the experimentally observed ones (see Tables 2-4), whereas for reductions close to $48 \%$ this approximation ensures a very accurate estimate of $\rho$.

Table 4. Dislocation densities $\rho \times 10^{14} \mathrm{~m}^{-2}$ computed by various models.

\begin{tabular}{ccc}
\hline Sample & K-E & Modified K-E \\
\hline B & 0.14 & 0.14 \\
C & 0.46 & 0.46 \\
D & 0.62 & 0.63 \\
E & 0.90 & 0.90 \\
F & 1.59 & 1.59 \\
\hline
\end{tabular}




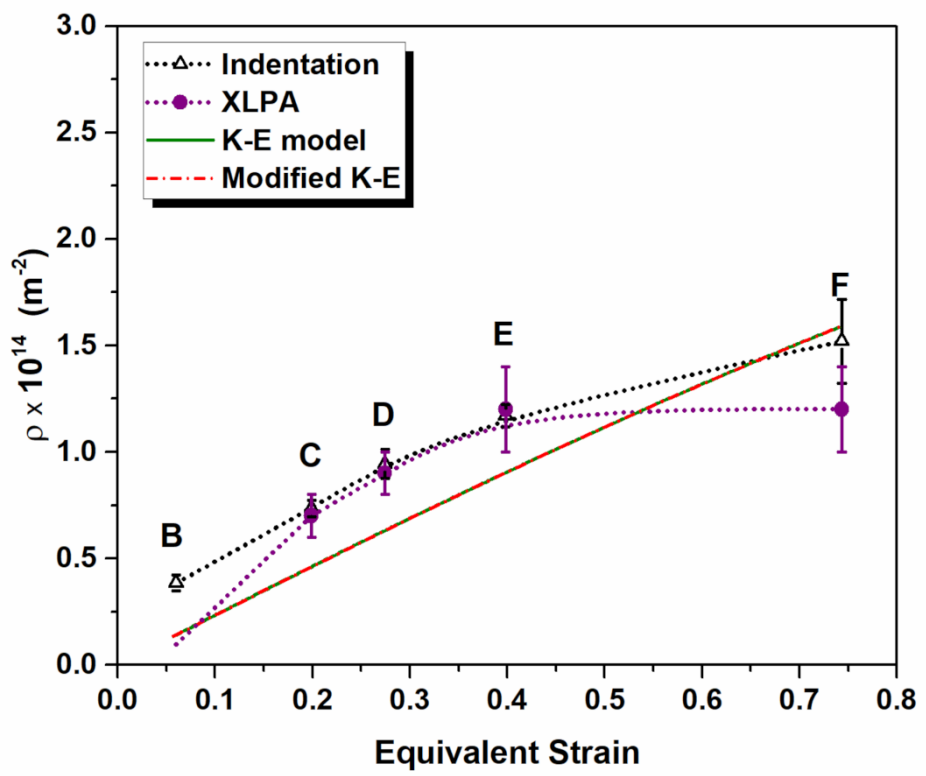

Figure 9. Evolution of dislocation density in $1050 \mathrm{Al}$ alloy defined by both experimental measurements and simulations.

Figure 10 shows the contribution of individual components to the total dislocation density computed with the K-E approach. It is obvious that for small strains there is an order of magnitude difference between the amount of mobile and forest dislocations; however, this deviation tends to decline with the increase in straining level. A similar evolutionary tendency was predicted by the discrete dislocation dynamic simulations [42].

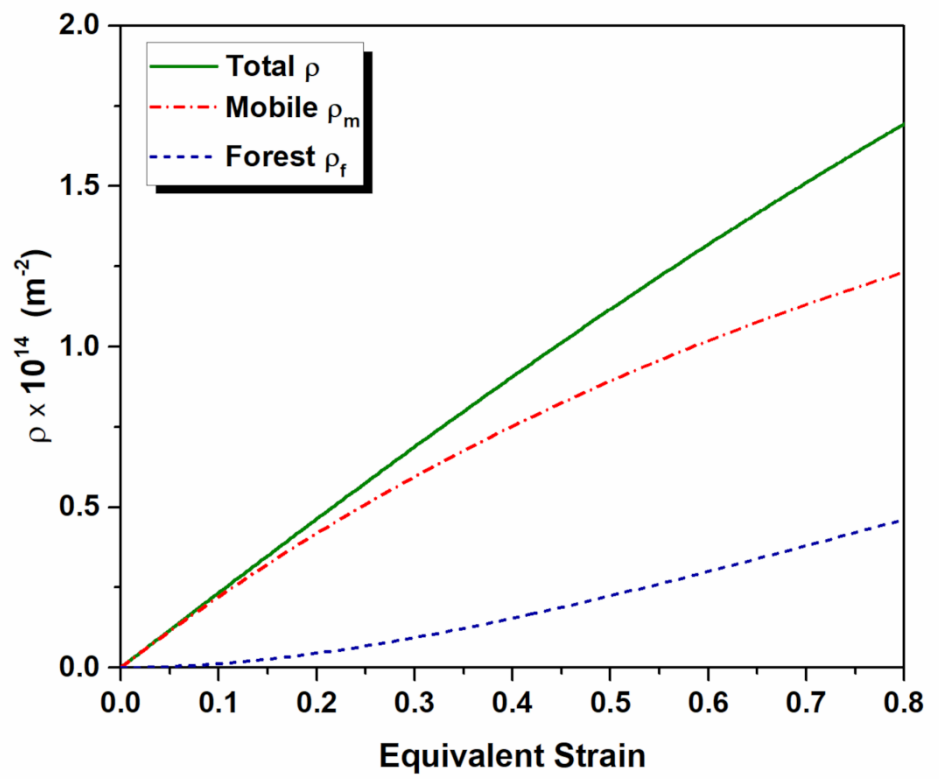

Figure 10. Contribution of the mobile and forest dislocations to the total dislocation density calculated by the K-E model [39].

The description of the evolution of dislocation density can also be conducted by the simplified K-E model, which was developed by Csanádi et al. [29,30]. The results of numerical simulations showed that for numerous metals [29,30], deformed at various temperatures, the model parameters $C_{2}$ and $C_{4}$ are nearly identical, and therefore, these two coefficients can be considered as equal quantities $\left(C_{2}=C_{4}\right)$. This simplification enables us to find the analytical solution (see Equation (6)) for the system of Equations (8) and 
(9). The simulation of dislocation density evolution by Equation (6) was performed with the $C_{1}=2.33 \times 10^{14} \mathrm{~m}^{-2}, C_{4}=1.15$ [30] and $\rho_{0}=\rho(\varepsilon=0)=10^{10} \mathrm{~m}^{-2}$. The simplified $\mathrm{K}-\mathrm{E}$ approximation (Equation (6)) provides nearly equal values, compared to the original $\mathrm{K}-\mathrm{E}$ model, as shown in Figure 9. The discrepancies observed between the experimental and simulated values can be explained by the fact that the $C_{i}$ coefficients found in the literature sources $[29,30]$ were defined for pure $\mathrm{Al}$, whereas the investigated $1050 \mathrm{Al}$ alloy can be considered as technically pure $\mathrm{Al}$ with traces of iron, which can affect the model parameters.

This study investigated the evolution of $\rho$ at relatively low deformation degrees $(\varepsilon<1)$, which provides a dislocation density of $\sim 10^{14} \mathrm{~m}^{-2}$; however, when higher strains are applied $(\varepsilon>3)$, the K-E-type models produce a saturation value of $\sim 3.95 \times 10^{14} \mathrm{~m}^{-2}$ (see Figure 11). This implies that in $\mathrm{Al}$ alloys with a low content of solutes, the rate of dislocation formation is high at lower strains and tends to level off at higher straining levels.

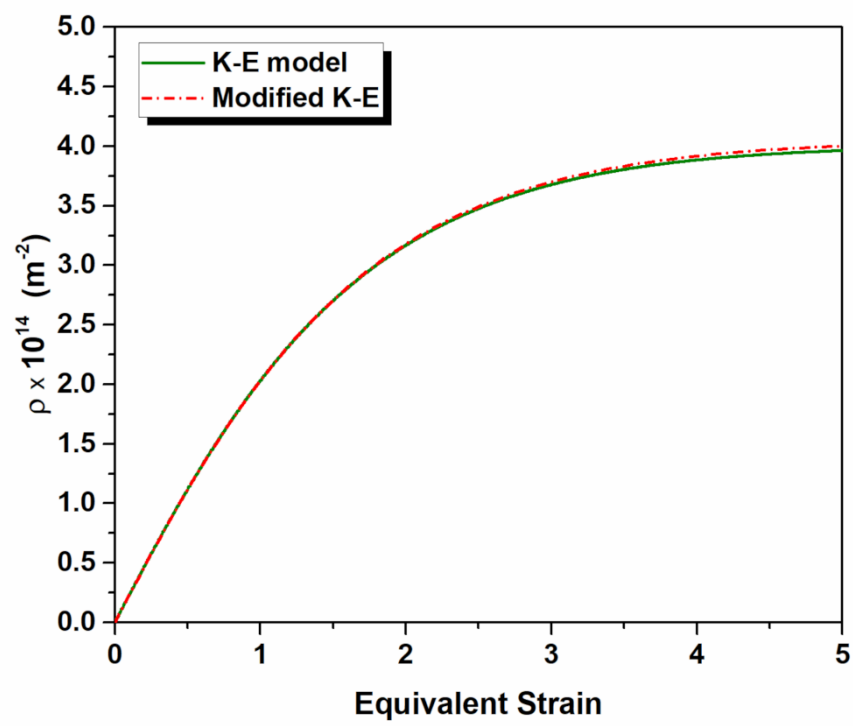

Figure 11. Results of simulations performed for Al with the Kubin-Estrin [39] and modified K-E [29] models.

\section{Conclusions}

The evolution of dislocation density during cold rolling can be successfully traced by both X-ray line profile analysis (XPLA) and microhardness indentation. Both methods provide comparable values for dislocation density; however, the indentation method tends to overestimate the quantity of dislocations at low deformations $(\sim 5 \%)$. Likewise, the indentation technique is not applicable for the assessment of dislocation quantity in the unstrained materials, and it leads to significantly high values. This is caused by the fact that microhardness testing may cause the formation of additional dislocations due to the deformation under the indenter. This effect is more pronounced for low strains when the material contains a very low density of dislocations.

Implementation of numerical approaches suggests that approximations like the KubinEstrin model or its simplified modification are capable of providing a reasonable estimate for the evolutionary pattern of dislocations. These models ensure a rational approximative value for the dislocation density at small and moderate reduction levels (5-30\%), while the discrepancy between the measured and simulated counterparts is negligible when the material is exposed to more severe straining $(\sim 47 \%)$.

Author Contributions: P.C. and J.J.S. contributed equally to this study. Conceptualization, J.J.S.; methodology, P.C., J.G., P.N., J.J.S.; investigation, P.C., J.G., P.N., J.J.S.; resources, J.J.S. and J.G.; 
modelling, J.G.B., J.J.S.; writing - review and editing, J.J.S., P.C., J.G.B., J.G., Q.X.; funding acquisition, J.J.S.; supervision, J.J.S. All authors have read and agreed to the published version of the manuscript.

Funding: This research was funded by the EFOP-3.6.1-16-2016-00018 project: "Improving the role of research + development + innovation in higher education through institutional developments assisting intelligent specialization in Sopron and Szombathely". The work was performed within the framework of the Széchenyi 2020 program: "Innovative processing technologies, applications of energy engineering, and implementation of wide-ranging techniques for microstructure investigation". This work was also completed as part of the ELTE Institutional Excellence Program (TKP2020-IKA-05) financed by the Hungarian Ministry of Human Capacities.

Institutional Review Board Statement: Not applicable.

Informed Consent Statement: Not applicable.

Data Availability Statement: The data presented in this study are available on request from the corresponding author. The data are not publicly available since they will be further postprocessed to develop new models.

Acknowledgments: Gyula Pál is gratefully acknowledged for their technical support and help in sample preparation.

Conflicts of Interest: The authors declare no conflict of interest.

\section{References}

1. Voyiadjis, G.Z.; Yaghoobi, M. Size Effects in Plasticity: From Macro to Nano; Elsevier: Amsterdam, The Netherland, 2020 ; p. 394.

2. Nye, J.F. Some geometrical relations in dislocated crystals. Acta Metall. 1953, 1, 153-162. [CrossRef]

3. Ashby, M.F. The deformation of plastically non-homogeneous materials. Phil. Mag. 1970, 21, 399-424. [CrossRef]

4. Humphreys, J.; Rohrer, G.S.; Rollett, A. Recrystallization and Related Annealing Phenomena, 3rd ed.; Elsevier: Amsterdam, The Netherland, 2017; p. 704.

5. Sidor, J.J.; Kestens, L.A.I. Analytical description of rolling textures in face-centred-cubic metals. Scripta Mater. 2013, 68, 273-276. [CrossRef]

6. Gubicza, J.; Ungár, T. Characterization of defect structures in nanocrystalline materials by x-ray line profile analysis. Z. Kristallographie 2007, 222, 567-579. [CrossRef]

7. Gubicza, J. Defect structure in nanomaterials; Woodhead Publishing: Sawston, UK, 2012; p. 358.

8. Gubicza, J. X-ray line profile analysis in Materials Science; IGI-Global: Hershey, PA, USA, 2014; p. 369.

9. Taheri, M.; Weiland, H.; Rollett, A. A method of measuring stored energy macroscopically using statistically stored dislocations in commercial purity aluminum. Metall. Mater. Trans. A 2006, 37, 19-25. [CrossRef]

10. Field, D.P.; Merriman, C.C.; Allain-Bonasso, N.; Wagner, F. Quantification of dislocation structure heterogeneity in deformed polycrystals by EBSD. Modelling Simul. Mater. Sci. Eng. 2012, 20, 024007. [CrossRef]

11. Ribárik, G.; Ungár, T.; Gubicza, J. MWP-fit: A program for multiple whole-profile fitting of diffraction peak profiles by ab initio theoretical functions. J. Appl. Cryst. 2001, 34, 669-676. [CrossRef]

12. Warren, B.E.; Averbach, B.L. The Effect of Cold-Work Distortion on X-Ray Patterns. J. Appl. Physics. 1950, 21, 595-599. [CrossRef]

13. Bertaut, E.F. Raies de Debye-Scherrer et repartition des dimensions des domains de Bragg dans les poudres polycrystallines. Acta Crystallographica 1950, 3, 14-18. [CrossRef]

14. Wilkens, M. The determination of density and distribution of dislocations in deformed single crystals from broadened X-ray diffraction profiles. Phys. Stat. Sol. 1970, 2, 359-370. [CrossRef]

15. Saleh, A.A.; Mannan, P.; Tome, C.N.; Pereloma, E.V. On the evolution and modelling of Cube texture during dynamic recrystallisation of Ni-30Fe-Nb-C model alloy. J. Alloys Compd. 2018, 748, 620-636. [CrossRef]

16. Durst, K.; Backes, B.; Göken, M. Indentation size effect in metallic materials: Correcting for the size of the plastic zone. Scr. Mater. 2005, 52, 1093-1097. [CrossRef]

17. Graça, S.; Colaço, R.; Carvalho, P.A.; Vilar, R. Determination of dislocation density from hardness measurements in metals. Mater Lett. 2008, 62, 3812-3814. [CrossRef]

18. Nix, W.D.; Gao, H. Indentation size effect in crystalline materials: A law for strain gradient plasticity. J. Mech. Phys. Solids 1998, 46, 411-425. [CrossRef]

19. Busby, J.T.; Hash, M.C.; Was, G.S. The relationship between hardness and yield stress in irradiated austenitic and ferritic steels. J. Nucl. Mater. 2005, 336, 267-278. [CrossRef]

20. Arsenlis, A.; Parks, D.M. Crystallographic aspects of geometrically necessary and statistically- stored dislocation density. Acta Mater. 1999, 47, 1597-1611. [CrossRef]

21. Gao, H.; Huang, Y. Geometrically necessary dislocation and size-dependent plasticity. Scr. Mater. 2003, 48, 113-118. [CrossRef]

22. Field, D.P.; Trivedi, P.B.; Wright, S.I.; Kumar, M. Analysis of local orientation gradients in deformed single crystals. Ultramicroscopy 2005, 103, 33-39. [CrossRef] 
23. Moussa, C.; Bernacki, M.; Besnard, R.; Bozzolo, N. About quantitative EBSD analysis of deformation and recovery substructures in pure Tantalum. 2015 IOP Conf. Ser. Mater. Sci. Eng. 2015, 89, 012038. [CrossRef]

24. Williamson, G.K.; Smallman, R.E. Dislocation Densities in Some Annealed and Cold-Worked Metals from Measurements on the X-ray Debye-Scherrer Spectrum. Philos. Mag. 1956, 1, 34-46. [CrossRef]

25. Smallman, R.E.; Westmacott, K.H. Stacking faults in face-centred cubic metals and alloys. Philos. Mag. 1957, 2, 669-683. [CrossRef]

26. Ma, K.; Wen, H.; Hu, T.; Topping, T.D.; Isheim, D.; Seidman, D.N.; Lavernia, E.J.; Schoenung, J.M. Mechanical behavior and strengthening mechanisms in ultrafine grain precipitation-strengthened aluminum alloy. Acta Mater. 2014, 62, 141-155. [CrossRef]

27. Zhao, Y.H.; Liao, X.Z.; Jin, Z.; Valiev, R.Z.; Zhu, Y.T. Microstructures and mechanical properties of ultrafine grained $7075 \mathrm{Al}$ alloy processed by ECAP and their evolutions during annealing. Acta Mater. 2004, 52, 4589-4599. [CrossRef]

28. Wang, X.; Guo, M.; Zhang, Y.; Xing, H.; Li, Y.; Luo, J.; Zhang, J.; Zhuang, L. The dependence of microstructure, texture evolution and mechanical properties of Al-Mg-Si-Cu alloy sheet on final cold rolling deformation. J. Alloys Compd. 2016, 657, 906-916. [CrossRef]

29. Csanádi, T.; Chinh, N.Q.; Gubicza, J.; Langdon, T.G. Plastic behavior of fcc metals over a wide range of strain: Macroscopic and microscopic descriptions and their relationship. Acta Mater. 2011, 59, 2385-2391. [CrossRef]

30. Csanádi, T.; Chinh, N.Q.; Gubicza, J.; Vörös, G.; Langdon, T.G. Characterization of stress-strain relationships in Al over a wide range of testing temperatures. Int. J. Plast. 2014, 54, 178-192. [CrossRef]

31. Pešička, J.; Kužel, R.; Dronhofer, A.; Eggeler, G. The evolution of dislocation density during heat treatment and creep of tempered martensite ferritic steels. Acta Mater. 2003, 51, 4847-4862. [CrossRef]

32. Sidor, J.J.; Petrov, R.H.; Kestens, L.A.I. Modeling the crystallographic texture changes in aluminum alloys during recrystallization. Acta Mater. 2011, 59, 5735-5748. [CrossRef]

33. Decroos, K.; Sidor, J.; Seefeldt, M. A new analytical approach for the velocity field in rolling processes and its application in through-thickness texture prediction. Metall. Mater. Trans. A 2014, 45, 948-961. [CrossRef]

34. Sidor, J.J. Assessment of Flow-Line Model in Rolling Texture Simulations. Metals 2019, 9, 1098. [CrossRef]

35. Groma, I.; Ungar, T.; Wilkens, M. Asymmetric X-ray Line Broadening of Plastically Deformed Crystals. I. Theory. J. Appl. Crystallogr. 1988, 21, 47-53. [CrossRef]

36. Gil Sevillano, J.; Van Houtte, P.; Aernoudt, E. Large Strain Work Hardening and Textures. Prog. Mater. Sci. 1980, 25, 69-412. [CrossRef]

37. Müllner, P.; Solenthaler, C. On the effect of deformation twinning on defect densities. Mater. Sci. Eng. A 1997, 230, 107-115. [CrossRef]

38. Nes, E. Modelling of work hardening and stress saturation in fcc metals. Prog. Mater. Sci. 1998, 41, 129-193. [CrossRef]

39. Kubin, L.P.; Estrin, Y. Evolution of dislocation densities and the critical conditions for the Portevin-Le Chatelier effect. Acta Metall. Mater. 1990, 38, 697-708. [CrossRef]

40. Mecking, H.; Kocks, U.F. Kinetics of flow and strain-hardening. Acta Met. 1981, 29, 1865-1875.

41. Estrin, Y.; Mecking, H. A unified phenomenological description of work hardening and creep based on one-parameter models. Acta Met. 1984, 32, 57-70. [CrossRef]

42. Wang, Z.Q.; Beyerlein, I.J.; Lesar, R. Plastic anisotropy in fcc single crystals in high rate deformation. Int. J. Plast. 2009, 25, 26-48. [CrossRef] 